\title{
Balancing outage performance of primary user and secondary user by relay-assisted primary transmission
}

\author{
Feng Zhao ${ }^{1 *}$, Xiangqi Sun ${ }^{1}$, Hongbin Chen ${ }^{1 *}$ and Rongfang Bie ${ }^{2}$
}

\begin{abstract}
In this paper, a cooperative transmission protocol for cognitive radio systems is proposed. In this protocol, the primary system comprises a transmitter (PT), a receiver (PR), and a decode-and-forward relay (Relay), while the secondary system comprises a transmitter (ST) and a receiver (SR). Both the ST and the Relay assist the transmissions of the primary users together. The outage probabilities of the primary system and the secondary system are analyzed and verified through simulations. In order to decrease outage probability of the secondary system, power allocation is performed at the ST. However, it will lead to deterioration of outage performance of the primary system. In order to guarantee outage performance of the primary system, a Relay is employed. Compared with two existing protocols, one without cooperation and the other with cooperation of the secondary system only, the proposed protocol is able to better balance outage performances of the primary system and the secondary system.
\end{abstract}

Keywords: Cognitive radio; Decode-and-forward relaying; Outage probability; Power allocation; Relay network; Spectrum sharing

\section{Introduction}

With the fast development of the telecommunications industry, wireless spectrum resources are becoming increasingly scarce. Because wireless spectrum resources are limited, improving spectral efficiency and allocating the spectrum resources efficiently become the ways to solve the problem. There are two kinds of users demanding spectrum with different priorities, which are licensed users and cognitive users. Licensed users, also called primary users, have a portion of licensed spectrum to transmit signals. However, cognitive users, known as secondary users, do not own licensed spectrum. As an effective approach to solve the problem of spectrum shortage, spectrum sharing [1], which allows a portion of secondary users to access the spectrum of primary users without harmful interference, was proposed to realize spectrum reuse. Compared with licensed spectrum, unlicensed spectrum is much less. Under these circumstances, cognitive radio [2] was proposed to improve the utilization of licensed spectrum.

\footnotetext{
* Correspondence: zhaofeng@guet.edu.cn; chbscut@163.com ${ }^{1}$ Key Laboratory of Cognitive Radio and Information Processing, Guilin University of Electronic Technology, Ministry of Education, Guilin 541004, China Full list of author information is available at the end of the article
}

Cognitive radio is an intelligent technology in spectrum sharing. In cognitive radio, secondary users are allowed to access the licensed spectrum on the condition that secondary users protect the transmissions of primary users to achieve spectrum sharing. In earlier works, the authors focused on some characteristics of a simple spectrum sharing protocol, such as achievable rate and outage performance, where the system consists of a primary and a secondary transmitter-receiver pairs [3-7]. In [6], the primary system transmitted the signal with the cooperation of the secondary system, and the outage performance of the primary system was improved obviously. A similar protocol with multiple antennas was considered in [8], where the achievable rate and bit error rate for arbitrary signal-to-noise ratio were analyzed. A protocol with selection of secondary users was considered in [9], where the outage probabilities of primary and secondary systems decrease as the number of secondary transmitters increases. Cooperative relaying technology was introduced into cognitive radio networks in order to enhance network capacity, scalability, and reliability of end-to-end communications. Though the performance of primary system is improved, the performance of secondary 
system may not be satisfied. In [10-13], the authors considered a protocol, where an intermediate relay cooperated with the communications between the secondary users, and they have considered the constraints on the average received interference at the primary users. The application of cooperative relays for secondary transmissions with the primary quality of service (QoS) constraint was considered in [14]. In $[15,16]$, the primary and secondary systems' outage performances and system capacities were studied with the same system model, respectively. Under the condition of keeping the normal transmissions of primary users, the performance of secondary system was ameliorated in [17-20]. In [21], a relay-assisted scheme was studied, where the relay helped the transmissions of the secondary users, considering interference from primary users. Cooperation diversity technology is able to reduce the effects of fading on signals in wireless communications, and relay selection can improve the achievable rate and reduce the sensitivity of channels at the destination node. Some protocols based on relay selection in cognitive radio were studied. The cooperation diversity and power allocation with optimal relay selection was considered in [22-25]. In [26], the authors proposed a relay-assisted system in cognitive radio, where the secondary transmitter and a relay competed for a licensed spectrum as long as the interference it incurs was not harmful, and the cooperative diversity gain in terms of outage performance grows as the number of relays increases. Spectrum sharing protocols based on amplify-and-forward relaying in Rayleigh and Nakagami- $m$ fading were studied in $[27,28]$, and the outage performances of the protocols based on relay selection in Rayleigh and Nakagami- $m$ fading were studied in $[29,30]$, respectively. In [31], the authors proposed a power allocation protocol for statistical QoS provisioning in multi-relay decode-and-forward cognitive networks. While these studies mainly considered perfect channel state information, the protocols considering imperfect channel state information have been taken into account. The secondary users' communications may cause harmful interference to the primary users if the channel state information of interference links is imperfect. The primary and secondary systems' outage performances with imperfect channel state information were studied in [32-34].

In the literature mentioned above, it is easy to find that the performances of the primary and secondary systems have been studied separately, but little literature has synthesized both of them to strike a balance. For example, in [6], the secondary system helps the transmissions of the primary users. Though the outage probability of the primary system is reduced, the outage performance of the secondary system may not be guaranteed. Motivated by this fact, we propose a spectrum sharing protocol for a cognitive relay network. This protocol consists of a primary system and a secondary system. The primary system consists of a primary transmitter (PT), a decode-andforward relay (Relay), and a primary receiver (PR). A secondary transmitter (ST) and a secondary receiver (SR) constitute the secondary system, which is allowed to access the licensed spectrum. In the proposed protocol, we ensure the outage probability of the primary system with the cooperation of the Relay and the secondary users. On the premise of smooth communications between the secondary users, we adjust the power allocation factor of the secondary system in order to improve the outage performance of the secondary system and maintain the outage performance of the primary system with the cooperation of the decode-and-forward relay.

The rest of this paper is organized as follows. In Section 2, a system model is introduced, where a secondary system and a relay cooperate with a primary system together. In Section 3, the transmission process of the proposed protocol is described, and the outage probabilities of the primary system and the secondary system are analyzed. Moreover, two existing protocols are reviewed for comparison. In Section 4, analytical results are verified through simulations, and effects of various parameters on outage probabilities are analyzed. Finally, some concluding remarks are made in Section 5.

\section{System model}

With the purpose of improving outage performances of the primary system and the secondary system, we consider a spectrum sharing system as depicted in Figure 1. The primary system comprises a PT, a PR, and a Relay. The secondary system comprises a ST and a SR. The secondary system and the Relay assist the transmissions of the primary users together. The outage performance

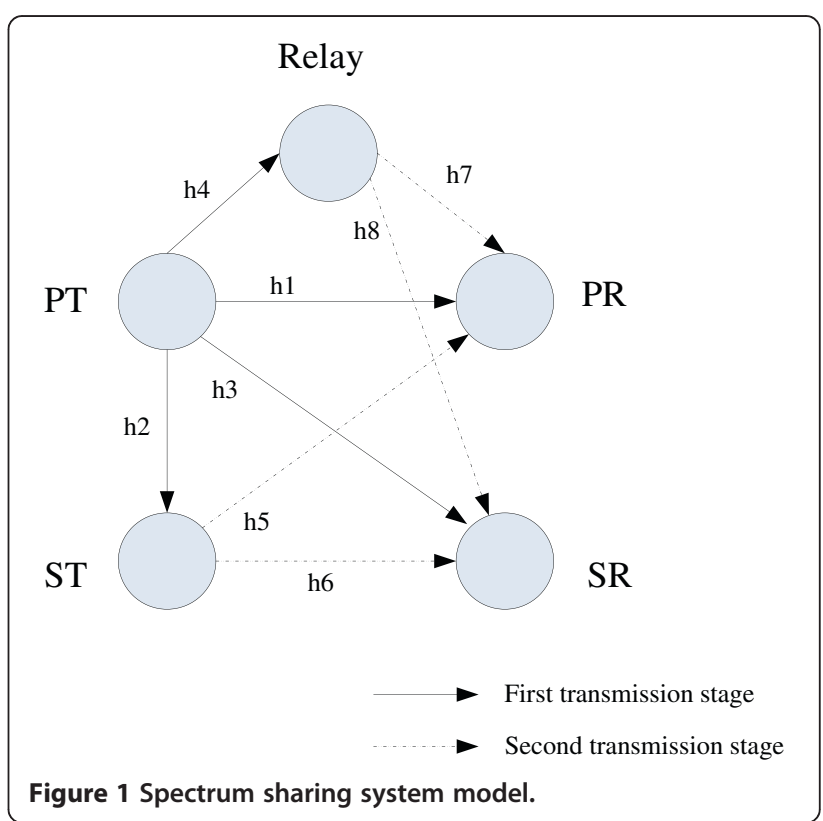


of the primary system benefits from the cooperation of the secondary users and the Relay. In this system, we adjust the power allocation factor at the ST in order to improve the outage performance of the secondary system, while its impact on the outage performance of the primary system will be compensated by the accommodation of the Relay.

The whole transmission process is divided into two stages. In the first transmission stage, the PT sends a primary signal to the PR, while the Relay and the ST also receive the signal. Then, the primary signal is decoded and superimposed at the ST, while the primary signal is decoded at the Relay. In the second transmission stage, a decoded signal and a weighted linear composite signal are transmitted by the Relay and the ST, respectively. The PR and the SR receive both of the signals sent from the Relay and the ST, respectively. At the PR, the primary signal is retrieved by a maximal-ratio combining (MRC) of the received signals from the two transmission stages. If the SR decodes the primary signal successfully, the primary signal will be removed as an interfering signal, and the secondary signal will be restored.

All the channels are assumed to experience Rayleigh fading [6]. The channel coefficients of the transmission links $\mathrm{PT} \rightarrow \mathrm{PR}, \mathrm{PT} \rightarrow \mathrm{ST}, \mathrm{PT} \rightarrow \mathrm{SR}, \mathrm{PT} \rightarrow$ Relay, $\mathrm{ST} \rightarrow$ $\mathrm{PR}, \mathrm{ST} \rightarrow \mathrm{SR}$, Relay $\rightarrow \mathrm{PR}$, and Relay $\rightarrow \mathrm{SR}$ are recorded by $h_{1}, h_{2}, h_{3}, h_{4}, h_{5}, h_{6}, h_{7}$, and $h_{8}$, respectively. Moreover, we assume $\mathrm{h}_{i} \sim C N\left(0, k_{i}^{-v}\right)[6], i=1,2,3,4,5,6,7$, and 8 , and it means that $h_{i}$ is a circularly symmetric complex Gaussian random variable with variance $k_{i}^{-v}$, where $k_{i}$ represents the normalized distance between two nodes, and $v$ represents the path loss exponent. That is to say, $k_{1}, k_{2}, k_{3}$, $k_{4}, k_{5}, k_{6}, k_{7}$, and $k_{8}$ denote the normalized distances between the PT and the PR, the PT and the ST, the PT and the SR, the PT and the Relay, the ST and the PR, the ST and the SR, the Relay and the PR, and Relay and the SR, respectively. This distance normalization is done with respect to the distance between the PT and the PR, i.e., $k_{1}=1$. Here, we also denote $\gamma_{i}=\left|h_{i}\right|^{2}$.

\section{Signal description and outage performance analysis}

3.1 Outage performance of the proposed protocol (scheme A) We study a two-stage transmission protocol, in which the secondary users and a relay assist the transmissions of the primary users together. As shown in Figure 1, solid lines and dotted lines represent the first transmission stage and the second transmission stage, respectively.

In the first transmission stage, the PT sends the primary signal $x_{\mathrm{p}}$, and the signal is received by the PR, ST, $\mathrm{SR}$, and Relay. We record the received signals by the PR, ST, SR, and Relay as $y_{11}, y_{21}, y_{31}$, and $y_{41}$, respectively. They can be represented by

$$
y_{a 1}=\sqrt{P_{\mathrm{p}}} h_{a} x_{\mathrm{p}}+n_{a 1}
$$

where $a=1,2,3,4$. Here, $P_{\mathrm{p}}$ is the transmission power of the primary user, $h_{a}$ is the channel coefficient, and $n_{a 1} \sim C N\left(0, \sigma^{2}\right)$ is an additive white Gaussian noise with zero mean and variance $\sigma^{2}$. The achievable rates between the PT and ST, the PT and SR, and the PT and Relay are denoted by $R_{2}, R_{3}$, and $R_{4}$, respectively. They can be written as

$$
R_{b}=\frac{1}{2} \log _{2}\left(1+\frac{P_{\mathrm{p}} \gamma_{b}}{\sigma^{2}}\right),
$$

where $b=2,3,4$. Here, the value $\frac{1}{2}$ is due to the fact that the whole transmission process is divided into two transmission stages. Then, both of the ST and Relay try to decode the received signal. At the ST, if decoding is successful, a weighted linear composite signal consisting of the primary signal and the secondary signal will be produced and transmitted in the second transmission stage. The regenerated signal is given by

$$
\chi_{\mathrm{r}}=\sqrt{\alpha P_{\mathrm{s}}} x_{\mathrm{p}}+\sqrt{(1-\alpha) P_{\mathrm{s}}} x_{\mathrm{s}}
$$

Here, $x_{\mathrm{s}}$ denotes the secondary signal, $P_{\mathrm{s}}$ is transmission power of the secondary transmitter, and $\alpha$ is the power allocation factor. If the ST fails to decode $x_{\mathrm{p}}$, it will keep silent in the second transmission stage. Likewise, if the Relay decodes $x_{\mathrm{p}}$ successfully, it will continue the transmissions in the next stage; otherwise, it will keep silent.

In the second transmission stage, $x_{\mathrm{r}}$ is transmitted by the ST and received by the PR and the SR. $x_{\mathrm{p}}$ is transmitted by the Relay and received by both of the PR and the SR. We represent the signal sent from the ST and received by the $\mathrm{PR}$ as $y_{12}$, which is given by

$$
\begin{aligned}
y_{12} & =h_{5} x_{\mathrm{r}}+n_{12} \\
& =\sqrt{\alpha P_{\mathrm{s}}} h_{5} x_{\mathrm{p}}+\sqrt{(1-\alpha) P_{\mathrm{s}}} h_{5} x_{\mathrm{s}}+n_{12}
\end{aligned}
$$

The signal sent from the Relay and received by the PR is denoted by $y_{22}$, which is given by

$$
y_{22}=\sqrt{P_{\mathrm{r}}} h_{7} x_{\mathrm{p}}+n_{22}
$$

The signal sent from the ST and received by the SR is denoted by $y_{32}$, which is given by

$$
\begin{aligned}
y_{32} & =h_{6} x_{\mathrm{r}}+n_{32} \\
& =\sqrt{\alpha P_{\mathrm{s}}} h_{6} x_{\mathrm{p}}+\sqrt{(1-\alpha) P_{\mathrm{s}}} h_{6} x_{\mathrm{s}}+n_{32}
\end{aligned}
$$

The signal sent from the Relay and received by the SR is denoted by $y_{42}$, which is given by

$$
y_{42}=\sqrt{P_{\mathrm{r}}} h_{8} x_{\mathrm{p}}+n_{42}
$$

Here, $P_{\mathrm{r}}$ is the transmission power of the Relay, and $n_{j 2}(j=1,2,3,4)$ is an additive white Gaussian noise with zero mean and variance $\sigma^{2}$. 
The transmission will be interrupted when the achievable rate is lower than the target rate. In the proposed protocol, the primary system will communicate normally in the following conditions: on one hand, if either the ST or the Relay is able to decode $x_{\mathrm{p}}$ successfully, $x_{\mathrm{p}}$ is sent through the transmission link where $x_{\mathrm{p}}$ is decoded successfully; on the other hand, if neither the ST nor the Relay decodes $x_{\mathrm{p}}$ successfully, $x_{\mathrm{p}}$ is sent through the direct link from the PT to the PR. In each condition, a MRC of the received signals is adopted to retrieve the primary signal in the second transmission stage [6]. In the following, we analyze the achievable rate for each of them.

(1) A case that the ST decodes the primary signal $x_{\mathrm{p}}$ successfully, but the Relay fails to decode it. In this case, $y_{11}$ and $y_{12}$ are combined with MRC at the PR. The achievable rate between the PT and the PR is calculated by

$$
R_{11}^{\mathrm{MRC}}=\frac{1}{2} \log _{2}\left(1+\frac{P_{\mathrm{p}} \gamma_{1}}{\sigma^{2}}+\frac{\alpha P_{\mathrm{s}} \gamma_{5}}{(1-\alpha) P_{\mathrm{s}} \gamma_{5}+\sigma^{2}}\right)
$$

(2) A case that both the ST and the Relay decode the primary signal successfully. Under the circumstances, $y_{11}, y_{12}$, and $y_{22}$ are combined with MRC at the PR. The achievable rate between the PT and the PR is calculated by

$$
R_{12}^{\mathrm{MRC}}=\frac{1}{2} \log _{2}\left(1+\frac{P_{\mathrm{p}} \gamma_{1}}{\sigma^{2}}+\frac{\alpha P_{\mathrm{s}} \gamma_{5}}{(1-\alpha) P_{\mathrm{s}} \gamma_{5}+\sigma^{2}}+\frac{P_{\mathrm{r}} \gamma_{7}}{\sigma^{2}}\right)
$$

(3) A case that the Relay is able to decode the primary signal successfully, but the ST fails to do so. Similarly, MRC will be applied to combine the signals $y_{11}$ and $y_{22}$ at the PR. The achievable rate between the PT and the $\mathrm{PR}$ is calculated by

$$
R_{13}^{\mathrm{MRC}}=\frac{1}{2} \log _{2}\left(1+\frac{P_{\mathrm{p}} \gamma_{1}}{\sigma^{2}}+\frac{P_{\mathrm{r}} \gamma_{7}}{\sigma^{2}}\right)
$$

(4) For the latter condition, neither the ST nor the Relay decodes the primary signal successfully. The primary signal $x_{\mathrm{p}}$ is transmitted through the direct link from the PT to the PR. The achievable rate between them is given by

$$
R_{1}=\log _{2}\left(1+\frac{P_{\mathrm{p}} \gamma_{1}}{\sigma^{2}}\right)
$$

So, the outage probability of the primary system with target rate $R_{\mathrm{pt}}$ is calculated by

$$
\begin{aligned}
P_{\text {out }}^{\mathrm{p}, \mathrm{A}} & =1-P_{\mathrm{r}}\left\{R_{2}>R_{\mathrm{pt}}\right\} P_{\mathrm{r}}\left\{R_{4}<R_{\mathrm{pt}}\right\} P_{\mathrm{r}}\left\{R_{11}^{\mathrm{MRC}}>R_{\mathrm{pt}}\right\} \\
& -P_{\mathrm{r}}\left\{R_{2}>R_{\mathrm{pt}}\right\} P_{\mathrm{r}}\left\{R_{4}>R_{\mathrm{pt}}\right\} P_{\mathrm{r}}\left\{R_{12}^{\mathrm{MRC}}>R_{\mathrm{pt}}\right\}, \\
& -P_{\mathrm{r}}\left\{R_{2}<R_{\mathrm{pt}}\right\} P_{\mathrm{r}}\left\{R_{4}>R_{\mathrm{pt}}\right\} P_{\mathrm{r}}\left\{R_{13}^{\mathrm{MRC}}>R_{\mathrm{pt}}\right\} \\
& -P_{\mathrm{r}}\left\{R_{2}<R_{\mathrm{pt}}\right\} P_{\mathrm{r}}\left\{R_{4}<R_{\mathrm{pt}}\right\} P_{\mathrm{r}}\left\{\frac{1}{2} R_{1}>R_{\mathrm{pt}}\right\}
\end{aligned}
$$

where the factor $\frac{1}{2}$ is due to the fact that the whole transmission process consists of two transmission stages. Since $\gamma_{1} \sim \varepsilon(1)$, and $\gamma_{i} \sim \varepsilon\left(k_{i}^{v}\right)(i=2,3,4,5,6,7)$, which means an exponential distributed random variable with mean $\frac{1}{k_{i}^{v}}$, we obtain

$$
\begin{aligned}
& P_{\mathrm{r}}\left\{R_{2}>R_{\mathrm{pt}}\right\}=P_{\mathrm{r}}\left\{\gamma_{2}>\frac{\sigma^{2}}{P_{\mathrm{p}}} \rho_{1}\right\}=\exp \left(-k_{2}^{v} \frac{\sigma^{2}}{P_{\mathrm{p}}} \rho_{1}\right) \\
& P_{\mathrm{r}}\left\{\frac{1}{2} R_{1}>R_{\mathrm{pt}}\right\}=P_{\mathrm{r}}\left\{\gamma_{1}>\frac{\sigma^{2}}{P_{\mathrm{p}}} \rho_{1}\right\}=\exp \left(-\frac{\sigma^{2}}{P_{\mathrm{p}}} \rho_{1}\right) \\
& P_{\mathrm{r}}\left\{R_{4}>R_{\mathrm{pt}}\right\}=P_{\mathrm{r}}\left\{\gamma_{4}>\frac{\sigma^{2}}{P_{\mathrm{p}}} \rho_{1}\right\}=\exp \left(-k_{4}^{v} \frac{\sigma^{2}}{P_{\mathrm{p}}} \rho_{1}\right)
\end{aligned}
$$

Here, $\rho_{1}=2^{2 R_{\mathrm{pt}}}-1$. Assuming that $P_{\mathrm{s}}>>\sigma^{2}$, we have

$$
\begin{aligned}
P_{\mathrm{r}}\left\{R_{11}^{\mathrm{MRC}}>R_{\mathrm{pt}}\right\} & =P_{\mathrm{r}}\left\{\frac{1}{2} \log _{2}\left(1+\frac{P_{\mathrm{p}} \gamma_{1}}{\sigma^{2}}+\frac{\alpha P_{\mathrm{s}} \gamma_{5}}{(1-\alpha) P_{\mathrm{s}} \gamma_{5}+\sigma^{2}}\right)>R_{\mathrm{pt}}\right\} \\
& \approx P_{\mathrm{r}}\left\{\frac{P_{\mathrm{p}} \gamma_{1}}{\sigma^{2}}+\frac{\alpha}{1-\alpha}>\rho_{1}\right\}=P_{\mathrm{r}}\left\{\gamma_{1}>\frac{\sigma^{2}}{P_{\mathrm{p}}}\left(\rho_{1}-\frac{\alpha}{1-\alpha}\right)\right\} \\
& =\left\{\begin{array}{cl}
\exp \left(-\frac{\sigma^{2}}{P_{\mathrm{p}}}\left(\rho_{1}-\frac{\alpha}{1-\alpha}\right)\right) & 0 \leq \alpha<\alpha^{*} \\
1 & \alpha^{*} \leq \alpha<1,
\end{array}\right.
\end{aligned}
$$

where $\alpha^{*}=\frac{\rho_{1}}{1+\rho_{1}}$. Similarly, we have

$$
\begin{aligned}
P_{\mathrm{r}}\left\{R_{12}^{\mathrm{MRC}}\right. & \left.>R_{\mathrm{pt}}\right\}=P_{\mathrm{r}}\left\{\frac{1}{2} \log _{2}\left(1+\frac{P_{\mathrm{p}} \gamma_{1}}{\sigma^{2}}+\frac{\alpha P_{\mathrm{s}} \gamma_{5}}{(1-\alpha) P_{\mathrm{s}} \gamma_{5}+\sigma^{2}}+\frac{P_{\mathrm{r}} \gamma_{7}}{\sigma^{2}}\right)>R_{\mathrm{pt}}\right\} \\
& \approx P_{\mathrm{r}}\left\{P_{\mathrm{p}} \gamma_{1}+P_{\mathrm{r}} \gamma_{7}>\sigma^{2}\left(\rho_{1}-\frac{\alpha}{1-\alpha}\right)\right\}=\left\{\begin{array}{cc}
P_{1} & 0 \leq \alpha<\alpha^{*} \\
1 & \alpha^{*} \leq \alpha<1,
\end{array}\right.
\end{aligned}
$$

where $\quad P_{1}=\exp \left(-\frac{\sigma^{2}\left(\rho_{1}-\frac{\alpha}{1-\alpha}\right)}{P_{\mathrm{p}}}\right)-\left(-1+k_{7}^{v} \frac{P_{\mathrm{p}}}{P_{\mathrm{r}}}\right)^{-1} \exp$ $\left(-k_{7}^{\nu} \frac{\sigma^{2}\left(\rho_{1}-\frac{\alpha}{1-\alpha}\right)}{P_{\mathrm{r}}}\right)\left(1-\exp \left(\left(-1+k_{7}^{\nu} \frac{P_{\mathrm{p}}}{P_{\mathrm{r}}}\right) \frac{\sigma^{2}\left(\rho_{1}-\frac{\alpha}{1-\alpha}\right)}{P_{\mathrm{p}}}\right)\right)$, and

$$
\begin{aligned}
P_{\mathrm{r}}\left\{R_{13}>R_{\mathrm{pt}}\right\}= & P_{\mathrm{r}}\left\{\frac{1}{2} \log _{2}\left(1+\frac{P_{\mathrm{p}} \gamma_{1}}{\sigma^{2}}+\frac{P_{\mathrm{r}} \gamma_{7}}{\sigma^{2}}\right)>R_{\mathrm{pt}}\right\} \\
= & P_{\mathrm{r}}\left\{P_{\mathrm{p}} \gamma_{1}+P_{\mathrm{r}} \gamma_{7}>\rho_{1} \sigma^{2}\right\}=1-P_{\mathrm{r}}\left\{P_{\mathrm{p}} \gamma_{1}+P_{r} \gamma_{7} \leq \rho_{1} \sigma^{2}\right\} \\
= & \exp \left(-\frac{\rho_{1} \sigma^{2}}{P_{\mathrm{p}}}\right)+\exp \left(-\frac{\rho_{1} \sigma^{2}}{P_{\mathrm{r}}} k_{7}^{v}\right)\left(-1+k_{7}^{v} \frac{P_{\mathrm{p}}}{P_{\mathrm{r}}}\right)^{-1} \\
& \left(\exp \left(\left(-1+k_{7}^{v} \frac{P_{\mathrm{p}}}{P_{\mathrm{r}}}\right) \frac{\rho_{1} \sigma^{2}}{P_{\mathrm{p}}}\right)-1\right)
\end{aligned}
$$

Substituting (13) to (18) into (12), we have

$$
P_{\text {out }}^{\mathrm{p}, \mathrm{A}}=\left\{\begin{array}{ll}
P_{\text {out }}^{\mathrm{p}, 1} & 0 \leq \alpha<\alpha * \\
P_{\text {out }}^{\mathrm{p}, 2} & \alpha^{*} \leq \alpha \leq 1
\end{array},\right.
$$

where 


$$
\begin{aligned}
& P_{\text {out }}^{\mathrm{p}, 1}=1-\exp \left(-k_{2}^{v} \frac{\sigma^{2}}{P_{\mathrm{p}}} \rho_{1}\right)\left(1-\exp \left(-k_{4}^{v} \frac{\sigma^{2}}{P_{\mathrm{p}}} \rho_{1}\right)\right) \exp \left(-\frac{\sigma^{2}}{P_{\mathrm{p}}}\left(\rho_{1}-\frac{\alpha}{1-\alpha}\right)\right)-\exp \left(-k_{2}^{v} \frac{\sigma^{2}}{P_{\mathrm{p}}} \rho_{1}\right) \exp \left(-k_{4}^{v} \frac{\sigma^{2}}{P_{\mathrm{p}}} \rho_{1}\right)\left(\begin{array}{c}
\exp \left(-\frac{\sigma^{2}\left(\rho_{1}-\frac{\alpha}{1-\alpha}\right)}{P_{\mathrm{p}}}\right)-\left(-1+k_{7}^{v} \frac{P_{\mathrm{p}}}{P_{\mathrm{r}}}\right)^{-1} \exp \left(-k_{7}^{v} \frac{\sigma^{2}\left(\rho_{1}-\frac{\alpha}{1-\alpha}\right)}{P_{\mathrm{r}}}\right) \\
\left(1-\exp \left(\left(-1+k_{7}^{v} \frac{P_{\mathrm{p}}}{P_{\mathrm{r}}}\right) \frac{\sigma^{2}\left(\rho_{1}-\frac{\alpha}{1-\alpha}\right)}{P_{\mathrm{p}}}\right)\right)
\end{array}\right), \\
& -\left(1-\exp \left(-k_{2}^{v} \frac{\sigma^{2}}{P_{\mathrm{p}}} \rho_{1}\right)\right) \exp \left(-k_{4}^{v} \frac{\sigma^{2}}{P_{\mathrm{p}}} \rho_{1}\right)\left(\begin{array}{l}
\exp \left(-\frac{\rho_{1} \sigma^{2}}{P_{\mathrm{p}}}\right)+\exp \left(-\frac{\rho_{1} \sigma^{2}}{P_{\mathrm{r}}} k_{7}^{v}\right)\left(-1+k_{7}^{v} \frac{P_{\mathrm{p}}}{P_{\mathrm{r}}}\right)^{-1} \\
\left(\exp \left(\left(-1+k_{7}^{v} \frac{P_{\mathrm{p}}}{P_{\mathrm{r}}}\right) \frac{\rho_{1} \sigma^{2}}{P_{\mathrm{p}}}\right)-1\right)
\end{array}\right)-\left(1-\exp \left(-k_{2}^{v} \frac{\sigma^{2}}{P_{\mathrm{p}}} \rho_{1}\right)\right)\left(1-\exp \left(-k_{4}^{v} \frac{\sigma^{2}}{P_{\mathrm{p}}} \rho_{1}\right)\right) \exp \left(-\frac{\sigma^{2}}{P_{\mathrm{p}}} \rho_{1}\right)
\end{aligned}
$$

and

$$
\begin{aligned}
P_{\text {out }}^{\mathrm{p}, 2}=1 & -\exp \left(-k_{2}^{v} \frac{\sigma^{2}}{P_{\mathrm{p}}} \rho_{1}\right)\left(1-\exp \left(-k_{4}^{v} \frac{\sigma^{2}}{P_{\mathrm{p}}} \rho_{1}\right)\right)-\exp \left(-k_{2}^{v} \frac{\sigma^{2}}{P_{\mathrm{p}}} \rho_{1}\right) \\
& \exp \left(-k_{4}^{v} \frac{\sigma^{2}}{P_{\mathrm{p}}} \rho_{1}\right)-\left(1-\exp \left(-k_{2}^{v} \frac{\sigma^{2}}{P_{\mathrm{p}}} \rho_{1}\right)\right) \exp \left(-k_{4}^{v} \frac{\sigma^{2}}{P_{\mathrm{p}}} \rho_{1}\right) \\
& \left(\exp \left(-\frac{\rho_{1} \sigma^{2}}{P_{\mathrm{p}}}\right)+\left(-1+k_{7}^{v} \frac{P_{\mathrm{p}}}{P_{\mathrm{r}}}\right)^{-1}\left(\exp \left(-\frac{\rho_{1} \sigma^{2}}{P_{\mathrm{p}}}\right)-\exp \left(-\frac{\rho_{1} \sigma^{2}}{P_{\mathrm{p}}} k_{7}^{v}\right)\right)\right) \\
& -\left(1-\exp \left(-k_{2}^{v} \frac{\sigma^{2}}{P_{\mathrm{p}}} \rho_{1}\right)\right)\left(1-\exp \left(-k_{4}^{v} \frac{\sigma^{2}}{P_{\mathrm{p}}} \rho_{1}\right)\right) \exp \left(-\frac{\sigma^{2}}{P_{\mathrm{p}}} \rho_{1}\right)
\end{aligned}
$$

The secondary signal is transmitted on the condition that both the ST and the SR decode the primary signal $x_{\mathrm{p}}$ successfully. In this case, the primary signal will be removed as an interference at the SR [6]. Therefore, the components $\sqrt{\alpha P_{\mathrm{s}}} h_{6} x_{\mathrm{p}}$ and $\sqrt{P_{\mathrm{r}}} h_{8} x_{\mathrm{p}}$ can be removed from (6) and (7), respectively. Thus, we have $\hat{y}_{32}=\sqrt{(1-\alpha) P_{\mathrm{s}}} h_{6}$ $x_{\mathrm{s}}+n_{32}$ and $\hat{y}_{42}=n_{42}$. So, the secondary signals will be transmitted in two cases, and they are as follows.

First, the Relay fails to decode $x_{\mathrm{p}}$, and it will keep silent in the second transmission stage. The achievable rate between the ST and the SR is calculated by

$$
R_{6}=\frac{1}{2} \log _{2}\left(1+\frac{(1-\alpha) P_{\mathrm{s}} \gamma_{6}}{\sigma^{2}}\right)
$$

Second, the Relay succeeds to decode $x_{\mathrm{p}}$, and it results in noise at the SR. Under the circumstances, the received signal at the $\mathrm{SR}$ is $y_{52}=\sqrt{(1-\alpha) P_{\mathrm{s}}} h_{6} x_{\mathrm{s}}+n_{32}$ $+n_{42}$. So, the achievable rate between the ST and the SR is calculated by

$$
R_{21}=\frac{1}{2} \log _{2}\left(\frac{(1-\alpha) P_{\mathrm{s}} \gamma_{6}}{2 \sigma^{2}}\right)
$$

So, we have

$$
\begin{aligned}
P_{\mathrm{out}}^{\mathrm{s}, \mathrm{A}}= & 1-P_{\mathrm{r}}\left\{R_{2}>R_{\mathrm{pt}}\right\} P_{\mathrm{r}}\left\{R_{3}>R_{\mathrm{pt}}\right\} P_{\mathrm{r}}\left\{R_{4}<R_{\mathrm{pt}}\right\} P_{\mathrm{r}}\left\{R_{6}>R_{\mathrm{st}}\right\} \\
& -P_{\mathrm{r}}\left\{R_{2}>R_{\mathrm{pt}}\right\} P_{\mathrm{r}}\left\{R_{3}>R_{\mathrm{pt}}\right\} P_{\mathrm{r}}\left\{R_{4}>R_{\mathrm{pt}}\right\} P_{\mathrm{r}}\left\{R_{21}>R_{\mathrm{st}}\right\} \\
& =1-\exp \left(-\left(k_{2}^{v}+k_{3}^{v}+k_{4}^{v}\right) \frac{\sigma^{2}}{P_{\mathrm{p}}} \rho_{1}-k_{6}^{v} \frac{2 \sigma^{2}}{(1-\alpha) P_{\mathrm{s}}} \rho_{3}\right) \\
& -\exp \left(-\left(k_{2}^{v}+k_{3}^{v}\right) \frac{\sigma^{2}}{P_{\mathrm{p}}} \rho_{1}-k_{6}^{v} \frac{\sigma^{2}}{(1-\alpha) P_{\mathrm{s}}} \rho_{3}\right)\left(1-\exp \left(-k_{4}^{v} \frac{\sigma^{2}}{P_{\mathrm{p}}} \rho_{1}\right)\right),
\end{aligned}
$$

where $\rho_{3}=2^{2 R_{\mathrm{st}}}-1$.

\subsection{Outage probability of the scheme without cooperation (scheme $B$ )}

The scheme without cooperation is the protocol without the secondary system and the Relay. It means that there is only a primary transmitter-receiver pair (PT-PR) in the system. In this system, the primary signal is transmitted only through the direct link from the PT to the PR. So, we obtain the outage probability of the primary system as follows:

$$
P_{\text {out }}^{0}=P_{\mathrm{r}}\left\{R_{1}<R_{\mathrm{pt}}\right\}=1-\exp \left(-\frac{\sigma^{2}}{P_{\mathrm{p}}} \rho_{2}\right),
$$

where $\rho_{2}=2^{R_{\mathrm{pt}}}-1$.

\subsection{Outage probability of the scheme with the} cooperation of the secondary system only (scheme C)

The scheme with the cooperation of the secondary system only is the protocol where the primary and secondary systems comprise a transmitter-receiver pair. In [6], this protocol has been analyzed thoroughly. The outage probability of the primary system is given by

$$
P_{\text {out }}^{\mathrm{p}, \mathrm{C}}= \begin{cases}P_{\text {out }}^{\mathrm{p}, 3} & 0 \leq \alpha<\alpha^{*} \\ P_{\text {out }}^{\mathrm{p}, 4} & \alpha^{*} \leq \alpha \leq 1\end{cases}
$$

where

$$
\begin{aligned}
& P_{\text {out }}^{\mathrm{p}, 3}=1-\exp \left(-\frac{\sigma^{2}}{P_{\mathrm{p}}}\left(\left(k_{2}^{v}+1\right) \rho_{1}-\frac{\alpha}{1-\alpha}\right)\right)-\exp \left(-\frac{\sigma^{2}}{P_{\mathrm{p}}} \rho_{1}\right) \\
& +\exp \left(-\frac{\sigma^{2}}{P_{\mathrm{p}}} \rho_{1}\left(k_{2}^{v}+1\right)\right) \text {, and } P_{\text {out }}^{\mathrm{p}, 4}=1-\exp \left(-k_{2}^{v} \frac{\sigma^{2}}{P_{\mathrm{p}}} \rho_{1}\right)- \\
& \exp \left(-\frac{\sigma^{2}}{P_{\mathrm{p}}} \rho_{1}\right)+\exp \left(-\frac{\sigma^{2}}{P_{\mathrm{p}}} \rho_{1}\left(k_{2}^{v}+1\right)\right) .
\end{aligned}
$$

The outage probability of the secondary system is given by

$$
\begin{aligned}
P_{\text {out }}^{\mathrm{s}, \mathrm{C}} & =1-P_{\mathrm{r}}\left\{R_{2}>R_{\mathrm{pt}}\right\} P_{\mathrm{r}}\left\{R_{3}>R_{\mathrm{pt}}\right\} P_{\mathrm{r}}\left\{R_{6}>R_{\mathrm{st}}\right\} \\
& =1-\exp \left(-\left(\frac{\sigma^{2}\left(k_{2}^{v}+k_{3}^{v}\right) \rho_{1}}{P_{\mathrm{p}}}+\frac{\sigma^{2} k_{6}^{v} \rho_{3}}{P_{\mathrm{s}}(1-\alpha)}\right)\right)
\end{aligned}
$$

\section{Simulation results and discussions}

In Section 3, we have introduced three different spectrum sharing schemes, including two kinds of cooperative transmission protocols and a protocol without 
cooperation. In this section, we will simulate $P_{\text {out }}^{\mathrm{p}}$ and $P_{\text {out }}^{\mathrm{s}}$ with the variation of $\alpha$ for each protocol. Most of the simulation parameters are the same as those in [6]. We set $v=4, \frac{P_{\mathrm{p}}}{\sigma^{2}}=\frac{P_{\mathrm{s}}}{\sigma^{2}}=\frac{P_{\mathrm{r}}}{\sigma^{2}}=20 \mathrm{~dB}$, and $\sigma^{2}=1$. Moreover, the target rates are set as $R_{\mathrm{pt}}=R_{\mathrm{st}}=2$, which are different from those in [6]. The reason is that the transmissions in the proposed scheme are able to support higher rates compared with those of the other two schemes. For ease of exposition, the topological structure is constructed like this as an example: the PT, PR, ST, SR, and Relay are collinear. In the two-dimensional plane, the PT and the PR are located at the points $(0,0)$ and $(1,0)$, respectively. The ST moves on the positive $X$ axis. The SR is at the midpoint of the PT and the ST, and the Relay is at the midpoint of the PT and the PR. In this topology, we have $k_{1}=1, k_{3}=\frac{1}{2} k_{2}, k_{4}=\frac{1}{2} k_{1}, k_{5}=\mid 1-$ $k_{2} \mid, k_{6}=\frac{1}{2} k_{2}, k_{7}=\frac{1}{2} k_{1}$, and $k_{8}=\frac{1}{2}\left|k_{1}-k_{2}\right|$. From the expression of $P_{\text {out }}^{\mathrm{p}, 1}$, it is easy to find that $\frac{\alpha}{1-\alpha}$ increases with the growth of $\alpha$. Furthermore, $P_{\text {out }}^{\mathrm{p}, 1}$ decreases with the growth of $\alpha$ in case of $0 \leq \alpha<\frac{\rho_{1}}{1+\rho_{1}}$. At the same time, we know that $P_{\text {out }}^{\mathrm{s}, \mathrm{A}}$ increases with the growth of $\alpha$ from (22). In other words, when more transmission power is allocated to assist the transmissions of the primary users at the ST, the outage performance of the primary system becomes better, whereas the outage performance of the secondary system becomes worse. In Figures 2 and 3, we plot the outage probabilities of the primary system and secondary system versus the power allocation factor when $k_{2}=0.5,1.2,1.92$, respectively. In the figures, lines represent theoretical results, and markers represent simulation results. Note that the theoretical results match with the simulation results very well. It is easy to find that in the proposed protocol, $P_{\text {out }}^{\mathrm{p}, \mathrm{A}}$ is lower than $P_{\text {out }}^{\mathrm{o}}$, which means that the outage probability of the primary system in scheme A is much lower than that in scheme B.

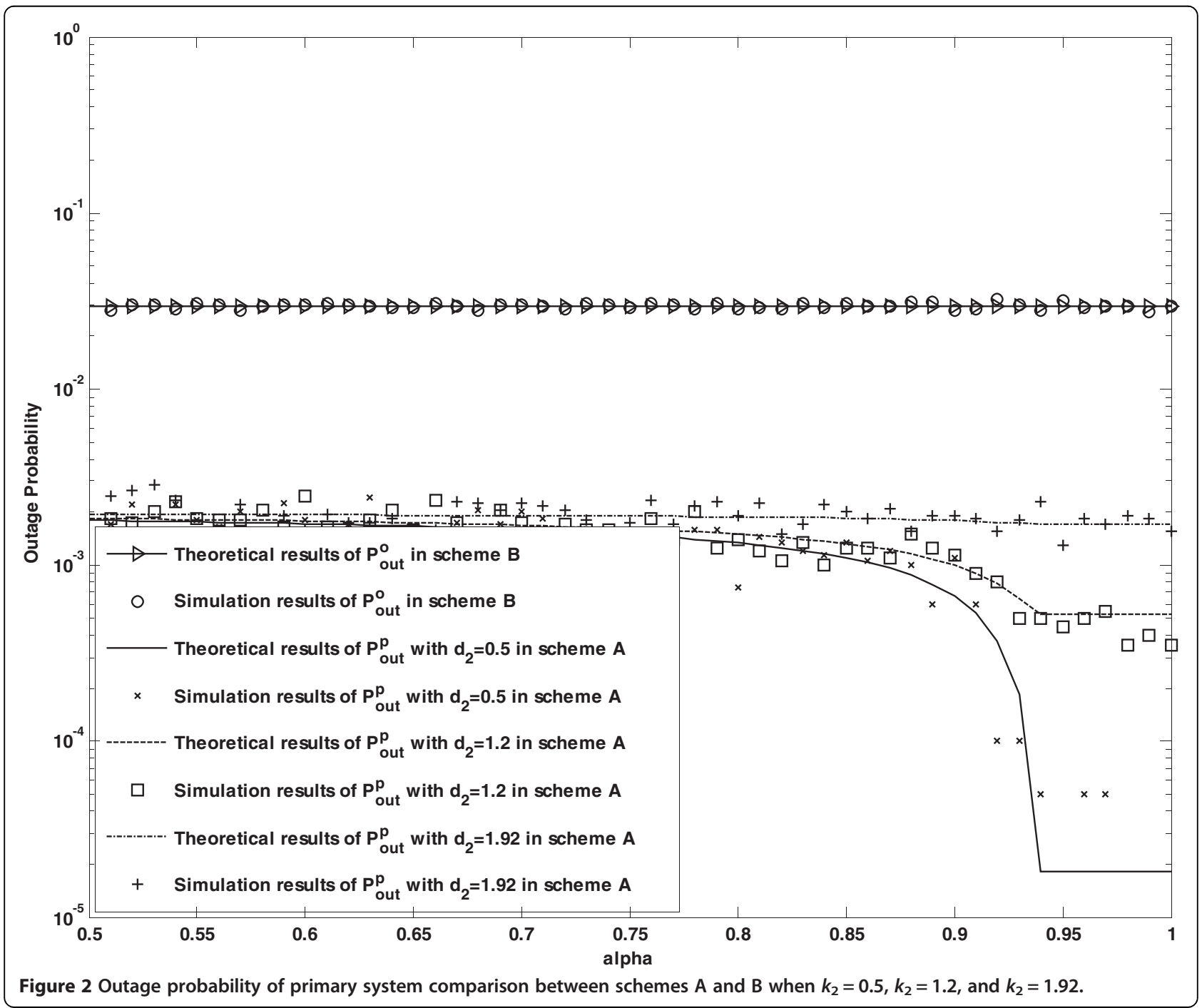




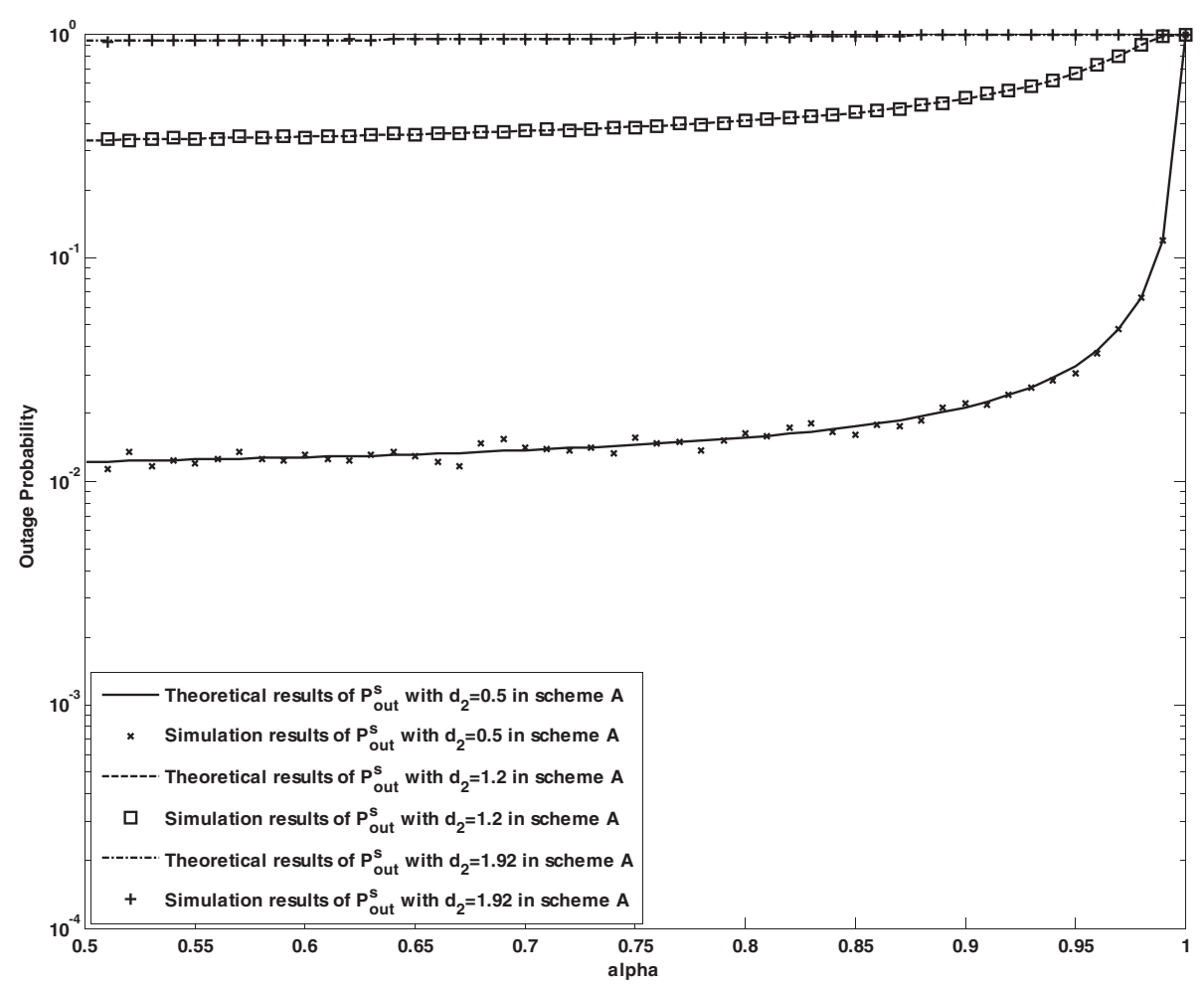

Figure 3 Outage probability of the secondary system in scheme $A$ when $k_{2}=0.5, k_{2}=1.2$, and $k_{2}=1.92$.

As shown in Figures 4, 5 and 6, we compare the outage performances in schemes $\mathrm{A}$ and $\mathrm{C}$ when $k_{2}=0.5, k_{2}=$ 1.2 , and $k_{2}=1.92$, respectively. In these figures, the topological structure is the same as that in Figure 2. The parameters are set as $v=4, \frac{P_{\mathrm{p}}}{\sigma^{2}}=\frac{P_{\mathrm{s}}}{\sigma^{2}}=\frac{P_{\mathrm{r}}}{\sigma^{2}}=20 \mathrm{~dB}, R_{\mathrm{pt}}=$ $R_{\mathrm{st}}=2$, and $\sigma^{2}=1$. The lines represent the theoretical results, and markers represent the simulation results. Compared with scheme $\mathrm{C}$, there is a decode-andforward relay cooperating with the primary users in scheme A, so the outage performance of the primary system will be improved. Because of the interference caused by the Relay, the outage performance of the secondary system becomes a little worse. From Figures 4, 5 and 6 , it is seen that with different settings of $k_{2}, P_{\text {out }}^{\mathrm{p}, \mathrm{A}}$ is less than $P_{\text {out }}^{\mathrm{o}}$ and far less than $P_{\text {out }}^{\mathrm{p}, \mathrm{C}}$ with the variation of $\alpha$.

We observe that the outage probabilities of the primary system in the three schemes are low, but the outage probabilities of the secondary system are high, especially in the setting of $k_{2}=1.92$. Since $P_{\text {out }}^{\mathrm{p}, \mathrm{A}}$ is less than $P_{\text {out }}^{\mathrm{o}}$, we are allowed to reduce the value of $\alpha$ in order to guarantee smooth communications between the secondary users. In other words, a small portion of transmission power of the secondary transmitter is allocated to send the primary signal. Under the circumstances, the outage performance of the primary system will degrade. Then, we try to guarantee $P_{\text {out }}^{\mathrm{p}, \mathrm{A}}$ by increasing the $P_{\mathrm{r}}$. So, we will simulate the influence of $P_{\mathrm{r}}$ on $P_{\text {out }}^{\mathrm{p}}$. Here, we set $k_{2}=1.92$, and $\alpha=0.1$. The other parameters except $P_{\mathrm{r}}$ are the same as those in Figure 6, which means that the ST is far away from the PT, and a small portion of the transmission power of the ST is allocated to send the primary signal. From Figure 7, we get that $P_{\text {out }}^{\mathrm{p}, \mathrm{A}}$ decreases with the growth of $P$ r, and $P_{\text {out }}^{\mathrm{p}, \mathrm{A}}$ tends to be stable with the growth of $P_{\mathrm{r}}$.

It is easy to find that both $P_{\text {out }}^{\mathrm{p}, \mathrm{A}}$ and $P_{\text {out }}^{\mathrm{o}}$ decrease with the growth of $P_{\mathrm{p}}$ from (19) and (23). From (22), we know that $P_{\text {out }}^{\mathrm{s}, \mathrm{A}}$ is independent from $P_{\mathrm{s}}$ and $k_{6}$ when $k_{6}$ is very small. That is to say that when the distance between the ST and the SR is short, $P_{\text {out }}^{\mathrm{s}, A}$ is not affected by $P_{\mathrm{s}}$ or $k_{6}$, and $P_{\text {out }}^{\mathrm{s}, \mathrm{A}}$ converges to $1-\exp \left(-\frac{\sigma^{2}\left(k_{2}^{v}+k_{3}^{v}\right) \rho_{1}}{P_{\mathrm{p}}}\right)$. Here, the topological structure is the same as that in Figure 2, and we set $P_{\mathrm{s}}=100, P_{\mathrm{r}}=10, \sigma^{2}=10, \alpha=0.5, R_{\mathrm{pt}}=R_{\mathrm{st}}=2$, and $v=4$. So, $P_{\text {out }}^{\mathrm{s}, \mathrm{A}}$ decreases with the growth of $P_{\mathrm{p}}$. From Figure 8 , we find that the simulation results agree with the analytical ones.

When the ST is not close to the SR, we analyze the outage probability expressions of the primary and secondary systems. For the primary system, $P_{\text {out }}^{\mathrm{p}, \mathrm{A}}$ is independent from $P_{\mathrm{s}}$ from (19), and $P_{\text {out }}^{\mathrm{s}, \mathrm{A}}$ decreases with the growth of $P_{\mathrm{s}}$ from (22). So, we build a different 


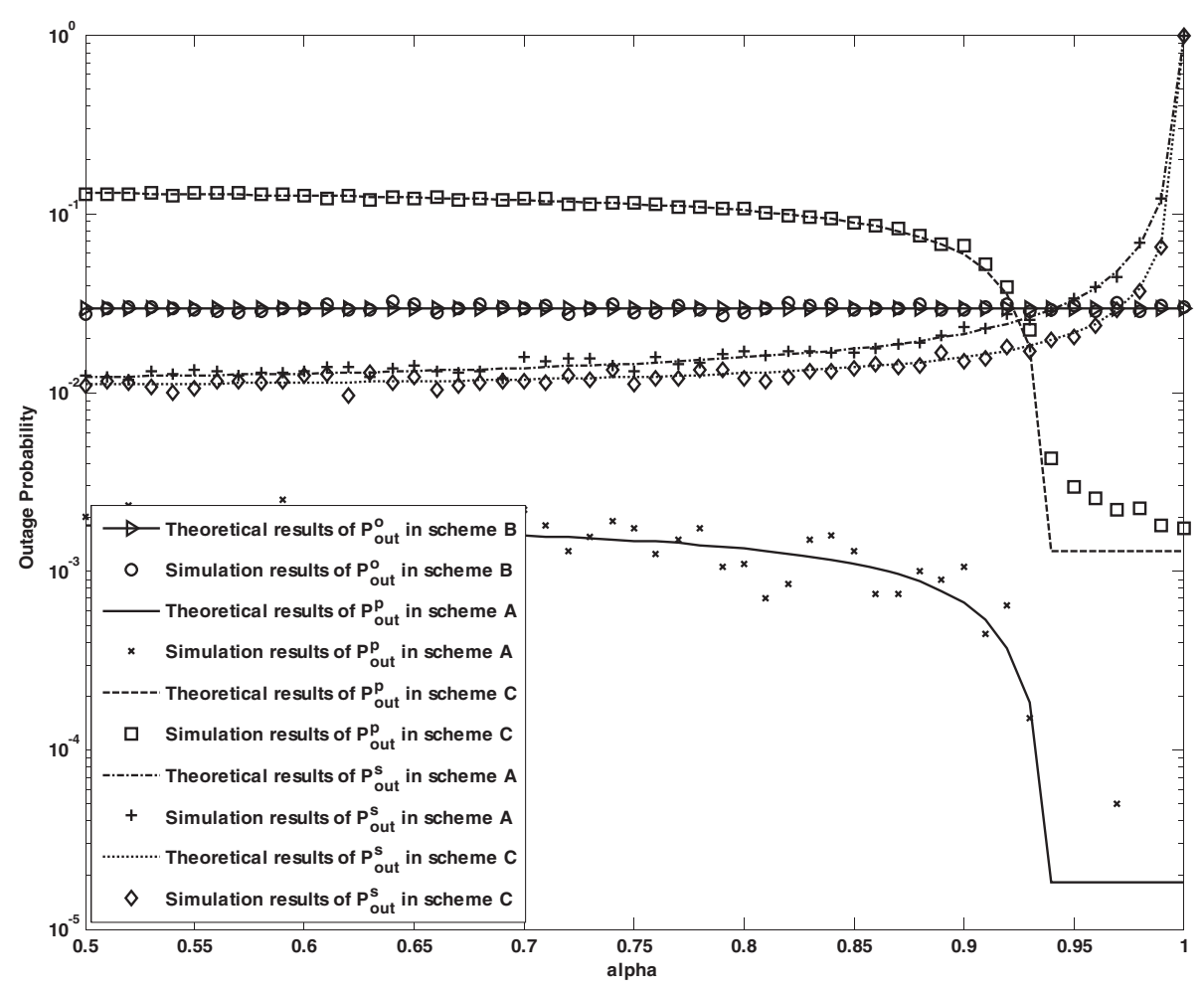

Figure 4 Outage probability comparison between schemes $A$ and $C$ when $k_{2}=0.5$.

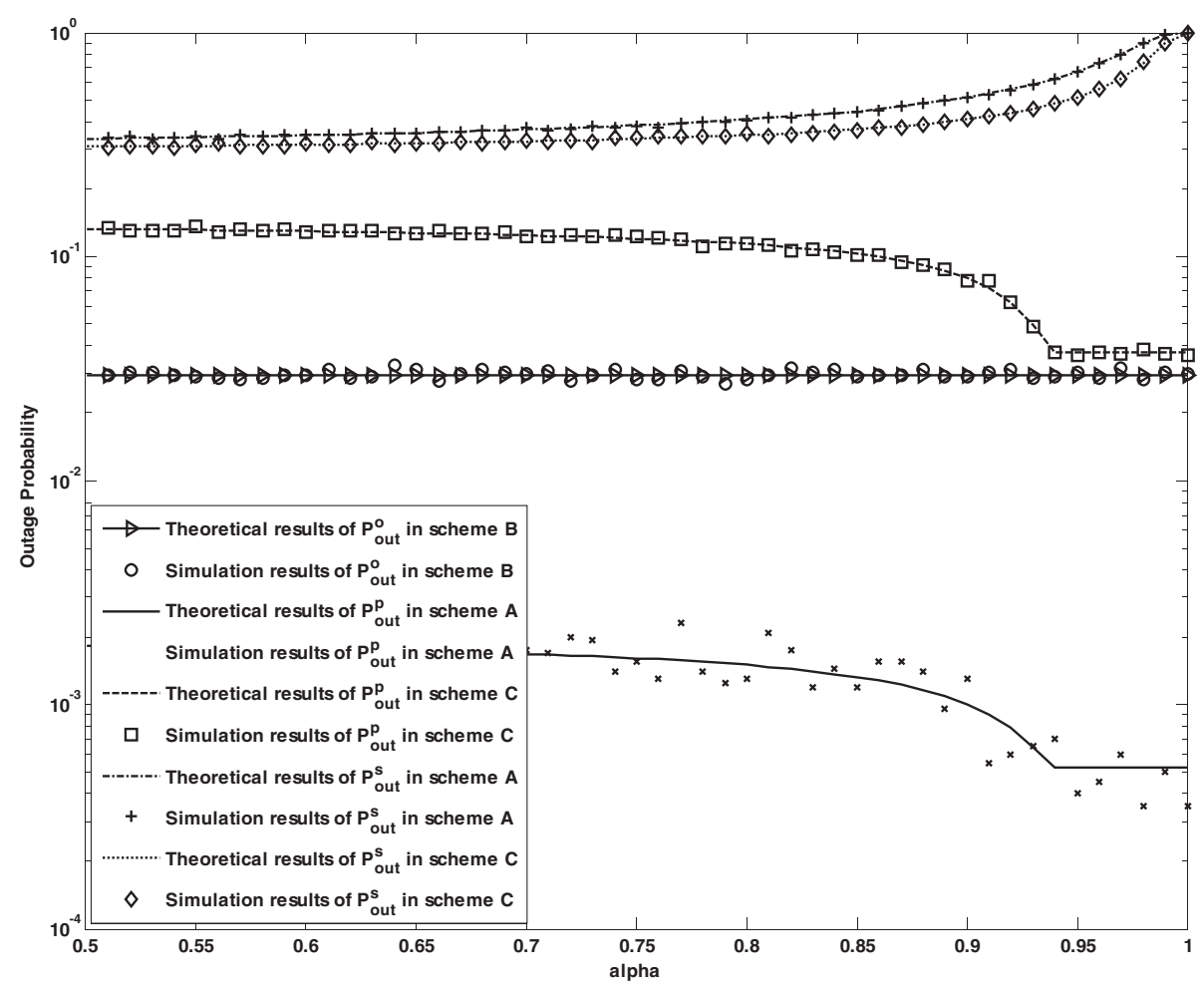

Figure 5 Outage probability comparison between schemes $\mathrm{A}$ and $\mathrm{C}$ when $k_{2}=1.2$. 


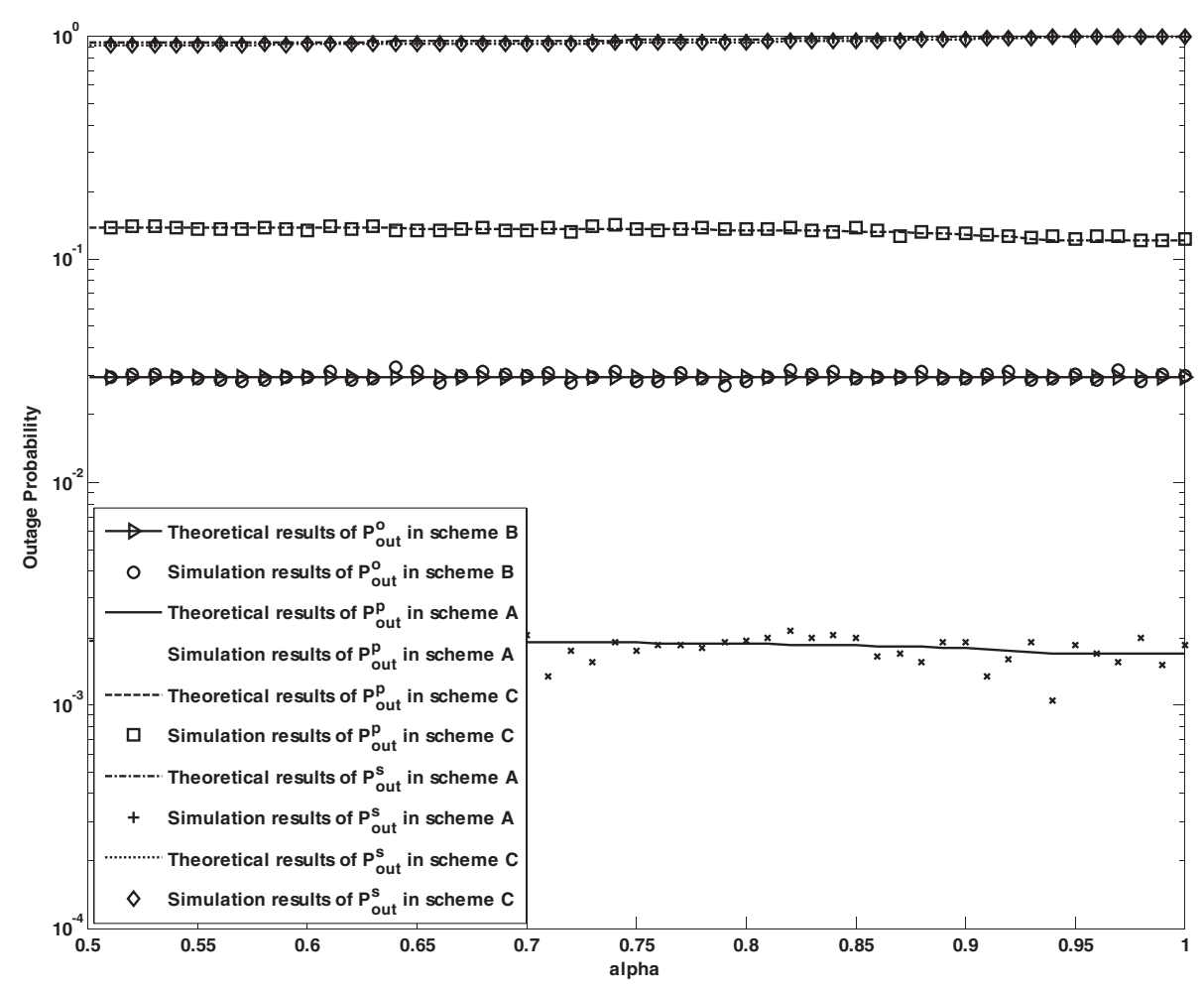

Figure 6 Outage probability comparison between schemes $A$ and $C$ when $k_{2}=1.92$.

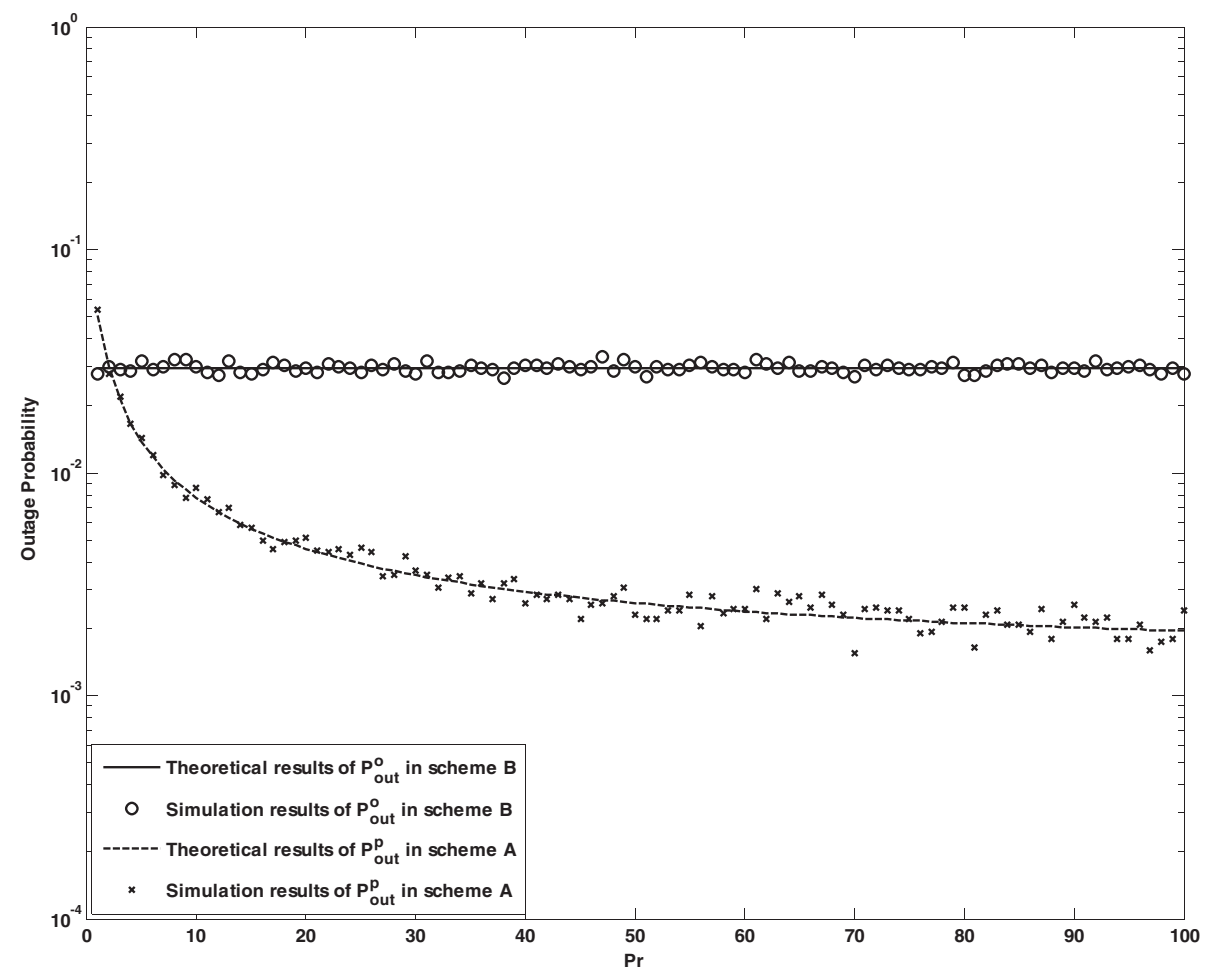

Figure 7 Outage probability of the primary system versus $P_{\mathrm{r}}$. 


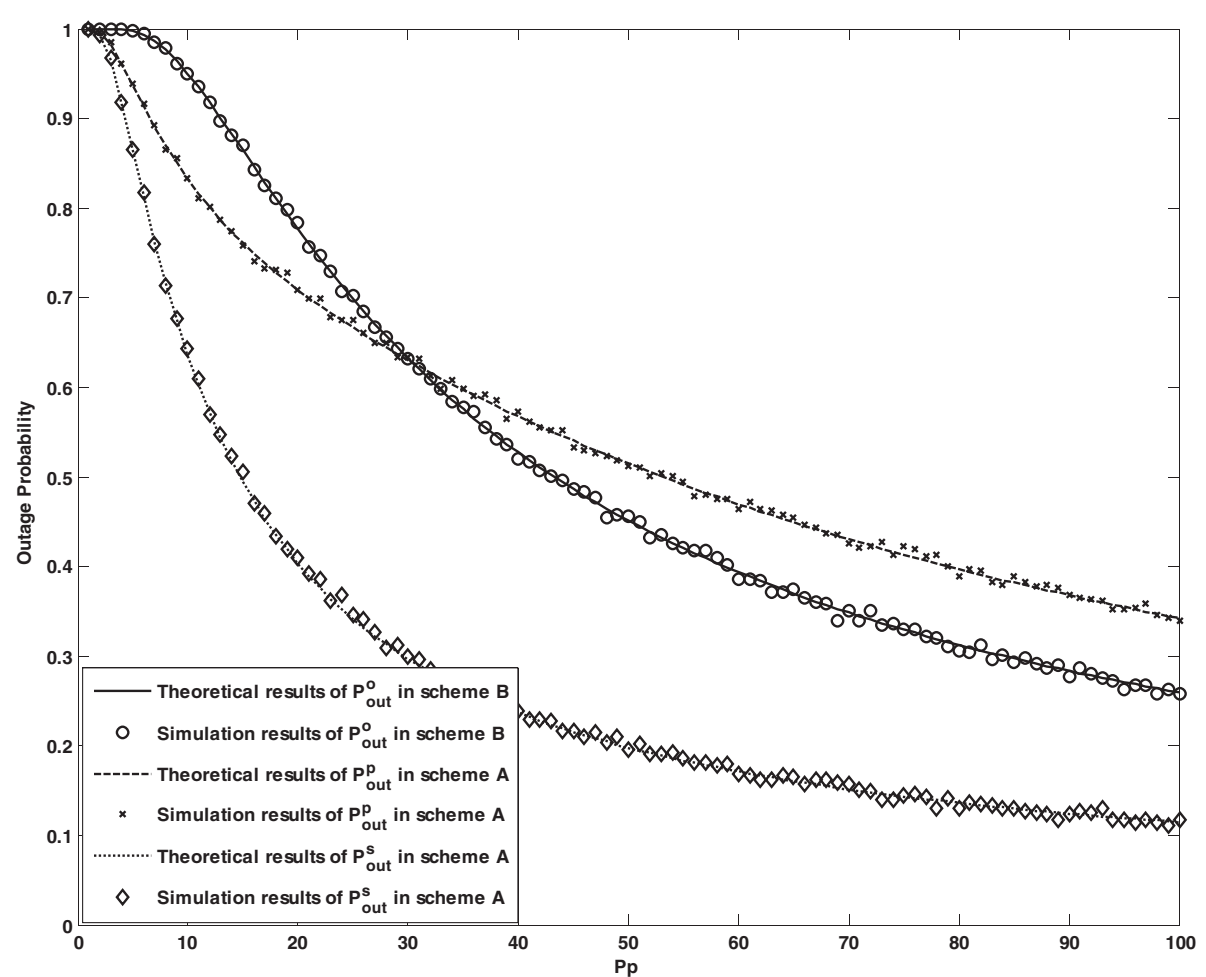

Figure 8 Outage probability versus $P_{\mathrm{p}}$.

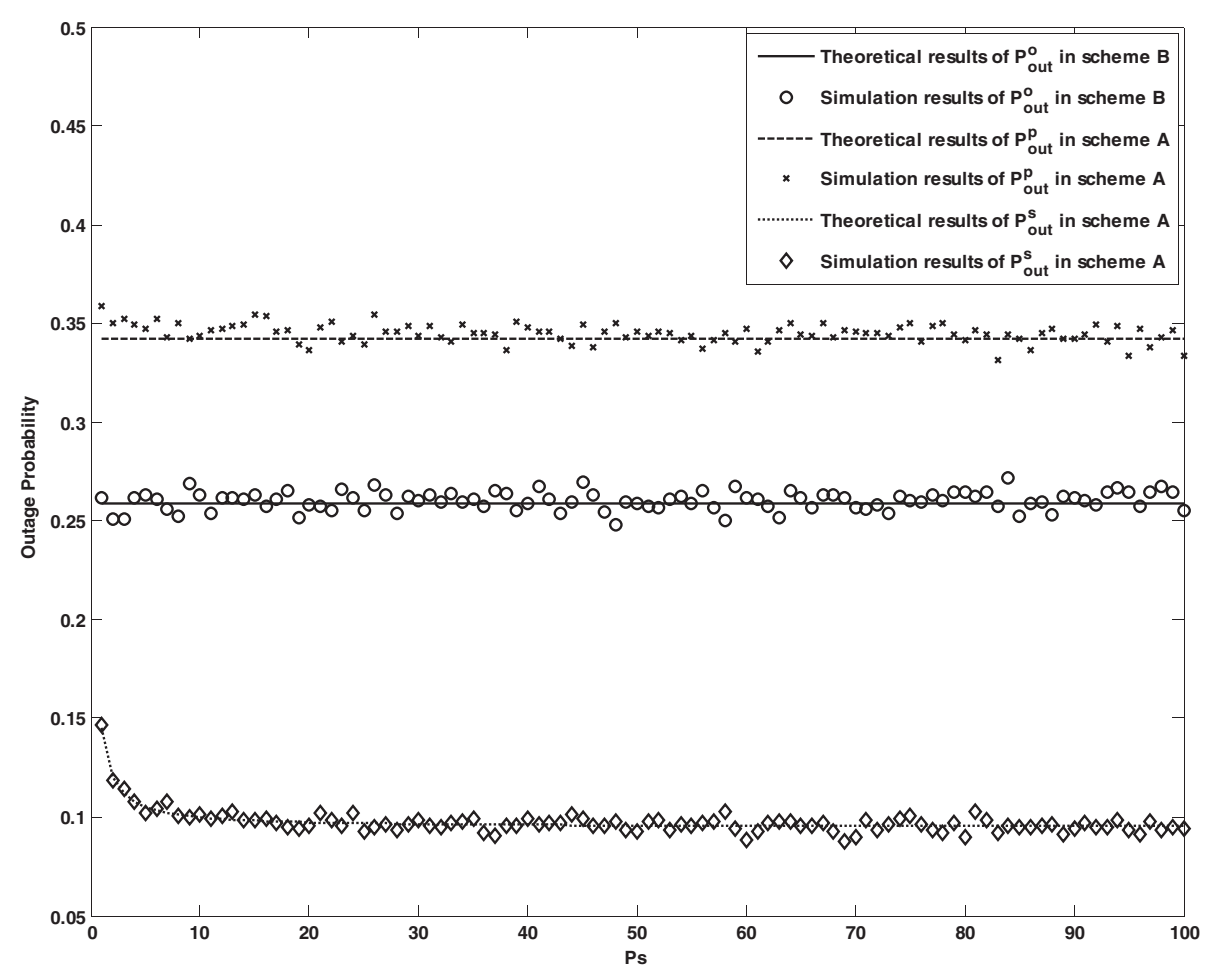

Figure 9 Outage probability versus $P_{\mathrm{s}}$. 
topological structure with the following parameters $k_{2}=$ $k_{3}=k_{4}=k_{5}=k_{6}=k_{7}=k_{8}=0.5, P_{\mathrm{p}}=100, P_{\mathrm{r}}=10, \sigma^{2}=10$, $\alpha=0.5, R_{\mathrm{pt}}=R_{\mathrm{st}}=2$, and $v=4$, which mean that the ST is far from the $\mathrm{SR}$, and the signal-to-noise ratios of the transmission links are relatively low. As Figure 9 shows, the variation of $P_{\text {out }}^{\mathrm{s}, \mathrm{A}}$ coincides with the analytical results. Though the growth of $P_{\mathrm{s}}$ improves the outage performance of the secondary system and does not greatly affect the outage performance of the primary system, $P_{\mathrm{s}}$ cannot increase unlimitedly. Meanwhile, the assistance of the Relay can improve the outage performance of the primary system.

\section{Conclusions}

In this paper, a cooperative transmission protocol where secondary users and a relay assist the transmissions of primary users was proposed. Compared with the protocol in [6], the proposed protocol can decrease the outage probability of the secondary system while maintaining the outage performance of the primary system. More specifically, the outage performance of the secondary system is improved by the power allocation at the secondary transmitter. Meanwhile, the outage performance of the primary system is guaranteed by the accommodating a relay.

\section{Competing interests}

The authors declare that they have no competing interests.

\begin{abstract}
Acknowledgements
We would like to thank the National Natural Science Foundation of China $(61172055,61162008)$, the Guangxi Natural Science Foundation (2011GXNSFB018072, 2013GXNSFGA019004), the Key Project of Chinese Ministry of Education (212131), the Foundation of Department of Education of Guangxi Province (201202ZD045), the Open Research Fund of Guangxi Key Laboratory of Wireless Wideband Communication \& Signal Processing (12106, 12103), and the Project of the Key Laboratory of Cognitive Radio and Information Processing (Guilin University of Electronic Technology), Ministry of Education (2013ZR02).
\end{abstract}

\section{Author details}

${ }^{1}$ Key Laboratory of Cognitive Radio and Information Processing, Guilin University of Electronic Technology, Ministry of Education, Guilin 541004, China. ${ }^{2}$ College of Information Science and Technology, Beijing Normal University, Beijing 100875, China.

Received: 23 December 2013 Accepted: 28 February 2014 Published: 17 March 2014

\section{References}

1. JM Peha, Approaches to spectrum sharing. IEEE Communications Magazine 43(2), 10-12 (2005)

2. J Mitola, Cognitive radio: making software radios more personal. IEEE Personal Communications 6(4), 13-18 (1999)

3. N Devroye, $\mathrm{P}$ Mitran, $\mathrm{V}$ Tarokh, Achievable rates in cognitive radio channels. IEEE Transactions on Information Theory 52(5), 1813-1827 (2006)

4. S Srinivasa, SA Jafar, Cognitive radios for dynamic spectrum access - the throughput potential of cognitive radio: a theoretical perspective. IEEE Communications Magazine 45(5), 73-79 (2007)

5. A Jovicic, P Viswanath, Cognitive radio: an information-theoretic perspective. IEEE Transactions on Information Theory 55(9), 3945-3958 (2009)
6. Y Han, A Pandharipande, S Ting, Cooperative decode-and-forward relaying for secondary spectrum access. IEEE Transactions on Wireless Communications 8(10), 4945-4950 (2009)

7. V Asghari, S Aïssa, Spectrum sharing in cognitive radio system: service-oriented capacity and power allocation. IET Communications 6(8), 889-899 (2010)

8. R Manna, RHY Louie, Y Li, B Vucetic, Cooperative spectrum sharing in cognitive radio networks with multiple antennas. IEEE Transactions on Signal Processing 59(11), 5509-5522 (2011)

9. Y Han, S Ting, A Pandharipande, Cooperative spectrum sharing protocol with secondary user selection. IEEE Transactions on Wireless Communications 9(9), 2914-2923 (2010)

10. Y Zou, Y Yao, B Zheng, Cognitive transmissions with multiple relays in cognitive radio networks. IEEE Transactions on Wireless Communications 10(2), 648-659 (2011)

11. S Sagong, J Lee, D Hong, Capacity of reactive DF scheme in cognitive relay networks. IEEE Transactions on Wireless Communications 10(10), 3133-3138 (2011)

12. V Asghari, S Aïssa, Performance of cooperative spectrum-sharing systems with amplify-and-forward relaying. IEEE Transactions on Wireless Communications 11(4), 1295-1300 (2012)

13. M Choi, J Park, S Choi, Simplified power allocation scheme for cognitive multi-node relay networks. IEEE Transactions on Wireless Communications 11(6), 2008-2012 (2012)

14. Y Zou, Y Yao, B Zheng, Cooperative relay techniques for cognitive radio systems: spectrum sensing and secondary user transmissions. IEEE Communications Magazine 50(4), 98-103 (2012)

15. J Si, Z Li, X Chen, B Hao, Z Liu, On the performance of cognitive relay networks under primary user's outage constraint. IEEE Communications Letters 15(4), 422-424 (2011)

16. J Si, Z Li, H Huang, J Chen, R Gao, Capacity analysis of cognitive relay networks with the PU's interference. IEEE Communications Letters 16(12), 2020-2023 (2012)

17. Z Yan, X Zhang, W Wang, Exact outage performance of cognitive relay networks with maximum transmit power limits. IEEE Communications Letters 15(12), 1317-1319 (2011)

18. V Asghari, S Aïssa, End-to-end performance of cooperative relaying in spectrum-sharing systems with quality of service requirements. IEEE Transactions on Vehicular Technology 60(6), 2656-2668 (2011)

19. J Lee, H Wang, JG Andrews, D Hong, Outage probability of cognitive relay networks with interference constraints. IEEE Transactions on Wireless Communications 10(2), 390-395 (2011)

20. W Chang, Cognitive radios for preserving primary outage performance over two-hop relay channels. IEEE Communications Letters 16(8), 1176-1179 (2012)

21. P Yang, L Luo, J Qin, Outage performance of cognitive relay networks with interference from primary user. IEEE Communications Letters 16(10), 1695-1698 (2012)

22. $Y$ Zou, J Zhu, B Zheng, Y Yao, An adaptive cooperation diversity scheme with best-relay selection in cognitive radio networks. IEEE Transactions on Signal Processing 58(10), 5438-5445 (2010)

23. L Li, X Zhou, H Xu, G Li, D Wang, A Soong, Simplified relay selection and power allocation in cooperative cognitive radio systems. IEEE Transactions on Wireless Communications 10(1), 33-36 (2011)

24. P Ubaidulla, S Aïssa, Optimal relay selection and power allocation for cognitive two-way relaying networks. IEEE Wireless Communications Letters 1(3), 225-228 (2012)

25. KB Fredj, S Aïssa, Performance of amplify-and-forward systems with partial relay selection under spectrum-sharing constraints. IEEE Transactions on Wireless Communications 11(2), 500-504 (2012)

26. Y Guo, G Kang, N Zhang, W Zhou, P Zhang, Outage performance of relay-assisted cognitive-radio system under spectrum-sharing constraints. Electronics Letters 46(2), 182-184 (2010)

27. VNQ Bao, TQ Duong, BD Costa, GC Alexandropoulos, A Nallanathan, Cognitive amplify-and-forward relaying with best relay selection in non-identical Rayleigh fading. IEEE Communications Letters 17(3), 475-478 (2013)

28. TQ Duong, DB Costa, M Elkashlan, VNQ Bao, Cognitive amplify-and-forward relay networks over Nakagami- $m$ fading. IEEE Transactions on Vehicular Technology 61(5), 2368-2374 (2012)

29. X Zhang, Z Yan, Y Gao, W Wang, On the study of outage performance for cognitive relay networks with the $N$ th best-relay selection in Rayleigh-fading channels. IEEE Wireless Communications Letters 2(1), 110-113 (2013) 
30. TQ Duong, DB Costa, TA Tsiftsis, C Zhong, A Nallanathan, Outage and diversity of cognitive relaying systems under spectrum sharing environments in Nakagami-m fading. IEEE Communications Letters 16(12), 2075-2078 (2012)

31. Y Wang, P Ren, $\mathrm{F}$ Gao, Power allocation for statistical QoS provisioning in opportunistic multi-relay DF cognitive networks. IEEE Signal Processing Letters 20(1), 43-46 (2013)

32. J Chen, J Si, Z Li, H Huang, On the performance of spectrum sharing cognitive relay networks with imperfect CSI. IEEE Communications Letters 16(7), 1002-1005 (2012)

33. SH Safavi, M Ardebilipour, S Salari, Relay beamforming in cognitive two-way networks with imperfect channel state information. IEEE Wireless Communications Letters 1(4), 344-347 (2012)

34. X Zhang, J Xing, Z Yan, Y Gao, W Wang, Outage performance study of cognitive relay networks with imperfect channel knowledge. IEEE Communications Letters 17(1), 27-30 (2013)

doi:10.1186/1687-6180-2014-34

Cite this article as: Zhao et al:: Balancing outage performance of primary user and secondary user by relay-assisted primary transmission. EURASIP Journal on Advances in Signal Processing 2014 2014:34.

\section{Submit your manuscript to a SpringerOpen ${ }^{\circ}$ journal and benefit from:}

- Convenient online submission

- Rigorous peer review

- Immediate publication on acceptance

- Open access: articles freely available online

- High visibility within the field

- Retaining the copyright to your article

Submit your next manuscript at $>$ springeropen.com 